\title{
Factors Affecting Exercise Self-efficacy in Predialysis Chronic Kidney Disease Patients
}

\author{
Zhang $\mathrm{F}^{*}$, Shen $\mathrm{Q}^{1}$, Zhang $\mathrm{W}^{1}$ and Zhou $\mathrm{W}^{2}$ \\ ${ }^{1}$ Nephrology Department, Longhua Hospital Affiliated to Shanghai University of Traditional Chinese Medicine, \\ Shang Hai, China \\ ${ }^{2}$ Department of Nursing, Longhua Hospital Affiliated to Shanghai University of Traditional Chinese Medicine, Shang \\ Hai, China
}

${ }^{*}$ Corresponding author: Zhang F, Nephrology Department, No. 725, Wan Ping Nan Road, Xuhui District, Shanghai 200032 P.R. China, Tel: 18817771780, E-mail: 1051071914@qq.com

Citation: Zhang F, Shen Q, Zhang W, Zhou W (2020) Factors Affecting Exercise Self-efficacy in Predialysis Chronic Kidney Disease Patients. J Nephrol Kidney Dis 1(1): 105. doi: 10.15744/2767-9225.1.105

Received Date: December 19, 2019 Accepted Date: April 28, 2020 Published Date: April 30, 2020

\begin{abstract}
Summary
Background: There was significant evidence to suggest that exercise improves physical function, symptom burden, and quality of life in predialysis chronic kidney disease (CKD) patients. However, most CKD patients cannot adherence to minimum recommended levels of exercise.

Objectives: To investigate the level and factors affecting of exercise self-efficacy in predialysis CKD patients.

Design: Exercise self-efficacy was measured using the Exercise Self-Efficacy Scale (ESES). Symptom burden was assessed using the Leicester Uraemic Symptom Score (LUSS).

Participants: A total of 137 predialysis CKD patients were recruited.

Measurements: Exercise self-efficacy of predialysis CKD patients was the main outcome measure.

Results: The mean score of exercise self-efficacy was $48.85 \pm 18.42$, at a moderate level. Multiple regression analysis showed that age, daily physical activity, and symptoms burden were influencing factors of exercise self-efficacy

Conclusion: The level of exercise self-efficacy of predialysis CKD patients needs to be improved urgently. Healthcare providers should pay special attention to elderly patients, and inform patients to increase their daily physical activity. Those with more complications should notice the management of their diseases to promote exercise.
\end{abstract}

Keywords: Predialysis; Chronic Kidney Disease; Exercise Self-efficac; Factors

\section{Introduction}

In China, there are about 100 million patients with CKD in different stages, and the prevalence rate of CKD in adults is about $11.8 \% \sim 13.0 \%$ $[1,2]$. CKD is increasingly becoming a worldwide health problem, and its drug treatment is becoming more and more mature. In recent years, it has been found that exercise can also control blood pressure and blood glucose; improve myocardial contractility and physical fitness [3]. However, CKD patients often lack proper exercise due to proteinuria, chronic malnutrition and falls.

\section{Literature Review}

CKD affects $8 \sim 16 \%$ of the world's population [4]. According to the report of the China Kidney Disease Network, the in-hospital mortality rate of CKD was $2.6 \%$, which was higher than that of patients without CKD (0.8\%) and with diabetes (1.5\%) [5]. In China, most CKD is caused by diabetic mellitus (27.0\%) and hypertension (20.8\%) [5]. Above-mentioned etiology repeated for a long period, leading to progression to end stage renal disease (ESRD), which necessitates treatment with renal replacement therapy such as transplant or dialysis, bring heavy burden to patients and families.

Previous studies found that the decline in physical function in patients with CKD began in the early stage of kidney disease (namely, without dialysis) and worsened with the loss of renal function. It is associated with increased risk of cardiovascular disease, muscle atrophy, decreased mobility and lower health-related quality of life [6]. In recent years, the benefits of exercise in improving the physical function of CKD patients have been increasingly recognized, including delaying access to dialysis [7]. However, the exercise compliance of predialysis CKD patients is only $40 \%$. 
Exercise self-efficacy refers to the confidence of individuals to complete a certain exercise behavior and achieve their goals through their ability [8]. Researches showed that improving the exercise self-efficacy of patients with chronic diseases can increase the amount of exercise [9,10]. However, at present, there are few studies on the level of exercise self-efficacy and its influencing factors in predialysis patients. Therefore, we performed the present study to describe the level of exercise self-efficacy and to determine the independent factors associated with exercise self-efficacy in predialysis CKD patients.

\section{Methods}

\section{Design}

A single-center cross-sectional study was carried out from October 2018 to April 2019. The specific aims of this study were as follows: (1) to describe exercise self-efficacy in predialysis CKD patients; (2) to explore the influence of socio-demographics, symptom burdens on exercise self-efficacy.

\section{Sample and setting}

The sample size was estimated using G-Power software (version 3.1) with an intermediate effect size of 0.15 , a significance level of 0.05 , power analysis of 0.08 , and 12 predictive variables. The sample size was increased by $20 \%$ to account for the non-response rate. 145 patients were selected using a convenience sample method. The inclusion criteria were as follows: (1) patients who met the diagnostic criteria of the National Kidney Foundation (K/DOQI) [11]; (2) patients who did not participate in other exercise-related clinical trials, and (3) patients who could communicate with each other in writing or language, had no mental and conscious disorders, and volunteered to participate in this study. Patients who received dialysis (ex. peritoneal dialysis, hemodialysis) or complicated with severe organic diseases will exclude.

\section{Data collection instrument}

Data were collected using a questionnaire containing three sections, a socio-demographic, the Leicester Uraemic Symptom Score (LUSS) and the Exercise Self-Efficacy Scale (ESES).

For socio-demographic, age, gender, body mass index (BMI), employment status, monthly average income, education level, stage of disease, time of diagnosis, comorbidity and daily physical activity were surveyed.

Symptom burden was measured by LUSS. The LUSS has proved useful and sensitive to change in predialysis CKD patients as an outcome measure to assess symptoms in cross-sectional studies [12], which assesses the intrusiveness (how much a symptom affects a patient's life) of 11 symptoms, including itching, sleep disturbance, loss of appetite, excessive tiredness, pain in bones/joints, poor concentration/mental alertness, impotence/lack of sex drive, loss of muscle strength/power, shortness of breath, muscle spasm/stiffness and restless legs. Intrusiveness was assessed with options of: "Not applicable", "Not at all intrusive", "Slightly intrusive", "Quite intrusive", "Very intrusive’ and "Extremely intrusive", scored from 0 to 5 [12].

ESES was used to measured exercise self-efficacy. The ESES instrument was developed by Bandura, with a Cronbach`s alpha coefficient 0.96 [13]. The ESES contains 18 items, each of which targets for situations that may affect the patient engage in regular exercise. The score of each item is 0-100, 0 means "not at all unconfident", 50 indicates "moderately confident", 100 indicates "highly confident". The scale consists of three dimensions, namely internal feeling subfactor, competing demands subfactor and situation/interpersonal subfactor. Total strength for each measure of self-efficacy is then calculated by summing the confidence ratings and dividing by the total number of items in the scale, resulting in a maximum possible efficacy score of 100 [14].

\section{Ethical considerations}

Before signing the consent form, each participant received an oral explanation of the study procedures, risks, and benefits of participation, and the right to withdraw at any time. Using identification numbers instead of real names for the data record protected participants' confidentiality.

\section{Statistical analysis}

Data were analyzed using the Statistical Package for Social Sciences, version 21.0 (SPSS Inc., Chicago, IL, USA). Descriptive analyses were used to describe the socio-demographic and study variables. Independent t-tests and one-way analyses of variance (ANOVA) were used to compare the exercise self-efficacy among the different socio-demographic groups. The Pearson correlation was used to calculate the relationship between exercise self-efficacy and symptom burden. Stepwise multiple regressions were used to determine the predictive factors of exercise self-efficacy. Categorical variables were dummy coded before analysis. A P value less than 0.05 was considered significant.

\section{Results}

\section{Participant's characteristics}

\begin{tabular}{|c|c|c|c|}
\hline \multicolumn{1}{|c|}{ Variables } & Frequency (\%) & Variables & Frequency (\%) \\
\hline Age (Year) & & Stage of disease & \\
\hline$<65$ & $94(68.6 \%)$ & CKD stage 1 & $30(21.9 \%)$ \\
\hline$\geq 65$ & $43(31.4 \%)$ & CKD stage 2 & $34(24.8 \%)$ \\
\hline Gender & & CKD stage 3 & $32(23.4 \%)$ \\
\hline Male & $78(56.9 \%)$ & CKD stage 4 & $20(14.6 \%)$ \\
\hline Female & $59(43.1 \%)$ & CKD stage 5 & $21(15.3 \%)$ \\
\hline
\end{tabular}




\begin{tabular}{|c|c|c|c|}
\hline Variables & Frequency (\%) & Variables & Frequency (\%) \\
\hline BMI $\left(\mathrm{kg} / \mathrm{m}^{2}\right)$ & & Time of diagnosis (year) & \\
\hline$<18.5$ & $6(4.4 \%)$ & $<1$ & $33(24.1 \%)$ \\
\hline $18.5 \sim 24$ & $66(48.2 \%)$ & $1 \sim 3$ & $19(13.9 \%)$ \\
\hline$>24$ & $65(47.4 \%)$ & $>3$ & $85(62.0 \%)$ \\
\hline Employment status & & Comorbidity & \\
\hline In business & $41(29.9 \%)$ & Cardiovascular diseases & $71(51.8 \%)$ \\
\hline Retire & $89(65.0 \%)$ & Diabetes & $39(28.5 \%)$ \\
\hline Unemployed & $7(5.1 \%)$ & Gout & $27(19.7 \%)$ \\
\hline Average monthly income & & Daily physical activity (min) & \\
\hline Poor & $12(8.8 \%)$ & $<30$ & $60(43.8 \%)$ \\
\hline Moderate & $88(64.2 \%)$ & $30 \sim 60$ & $50(36.5 \%)$ \\
\hline Good & $37(27.0 \%)$ & $>60$ & $27(19.7 \%)$ \\
\hline \multicolumn{4}{|l|}{ Education level } \\
\hline \multicolumn{4}{|l|}{ Primary school and } \\
\hline below & $21(15.3 \%)$ & & \\
\hline Junior middle school & $46(33.6 \%)$ & & \\
\hline Senior school or technical secondary school & $31(22.6 \%)$ & & \\
\hline College and above & $39(28.5 \%)$ & & \\
\hline
\end{tabular}

CKD: Chronic Kidney Disease; BMI: Body Mass Index

Table 1: Socio-demographics and clinical characteristics of participants $(n=137)$

137 patients participated in the current study giving a response rate of $94.48 \% .94$ (68.6\%) were less than 65 years and there were 78 (56.9\%) males and $59(43.1 \%)$ females. 66 (48.2\%) participants were normal weight. Only 41 (29.9\%) were still in business, and 37 (27.0\%) considered their monthly average income is good. The rest of the socio-demographic and clinical characteristics of the study participants are presented in Table 1.

\section{Exercise self-efficacy}

The participant's average score on exercise self-efficacy was $48.85 \pm 18.42$ (Range $=12.22-93.33$ ), indicating moderate exercise self-efficacy (Table 2). The score of internal feeling subfactor, competing demands subfactor and situation/interpersonal subfactor is $48.26 \pm 20.68$, $54.73 \pm 18.60$ and $43.56 \pm 18.31$, respectively. The score and sequencing for each item was shown in Table 2 .

\begin{tabular}{|l|c|c|}
\hline \multicolumn{1}{|c|}{ Item } & Score $(\overline{\mathbf{x}} \pm \mathbf{s})$ & Sequencing \\
\hline A total score of exercise self-efficacy & $\mathbf{4 8 . 8 5} \pm \mathbf{1 8 . 4 2}$ & - \\
\hline Internal feeling subfactor & $\mathbf{4 8 . 2 6 \pm 2 0 . 6 8}$ & - \\
\hline 1.When you are feeling tired & $39.12 \pm 25.01$ & 14 \\
\hline 2.When you are feeling under pressure from work & $52.92 \pm 23.71$ & 9 \\
\hline 3.When you are experiencing bad weather & $33.28 \pm 26.52$ & 16 \\
\hline 5.When you are experiencing personal problems & $53.87 \pm 20.59$ & 8 \\
\hline 6.When you are feeling depressed & $55.11 \pm 21.22$ & 7 \\
\hline 7.When you are feeling anxious & $55.26 \pm 21.49$ & 6 \\
\hline Competing demands subfactor & $\mathbf{5 4 . 7 3 \pm 1 8 . 6 0}$ & - \\
\hline 4.After recovering from an injury that caused you to stop exercising & $60.66 \pm 22.50$ & 5 \\
\hline 8.After recovering from an illness that caused you to stop exercising & $64.38 \pm 21.40$ & 3 \\
\hline 9.When you feel physical discomfort when you exercise & $28.18 \pm 23.40$ & 17 \\
\hline 10.After a vacation & $63.43 \pm 20.31$ & 4 \\
\hline 14.If you don't reach your exercise goals & $67.23 \pm 22.58$ & 2 \\
\hline 15.Without support from your family or friends & $44.49 \pm 22.12$ & 11 \\
\hline Situation/interpersonal subfactor & $\mathbf{4 3 . 5 6 \pm 1 8 . 3 1}$ & - \\
\hline 11.When you have too much work to do at home & $41.97 \pm 21.21$ & 13 \\
\hline 12.When visitors are present & $27.45 \pm 24.85$ & 18 \\
\hline 13.When there are other interesting things to do & $44.67 \pm 19.78$ & 10 \\
\hline
\end{tabular}




\begin{tabular}{|l|c|c|}
\hline 16.During a vacation & $67.95 \pm 20.94$ & 1 \\
\hline 17.When you have other time commitments & $36.79 \pm 21.32$ & 15 \\
\hline 18.After experiencing family problems & $42.55 \pm 20.69$ & 12 \\
\hline
\end{tabular}

Table 2: Score of exercise self-efficacy and each item $(n=137)$

\section{Factors associated exercise self-efficacy}

\begin{tabular}{|c|c|c|c|}
\hline Variables & A score of exercise self-efficacy & $t / F$ value & $P$ value \\
\hline \multicolumn{2}{|l|}{ Age (years) } & \multirow{3}{*}{1.988} & \multirow{3}{*}{0.049} \\
\hline$<65$ & $50.94 \pm 19.07$ & & \\
\hline$\geq 65$ & $44.28 \pm 16.18$ & & \\
\hline \multicolumn{2}{|l|}{ Gender } & \multirow{3}{*}{-2.310} & \multirow{3}{*}{0.022} \\
\hline Male & $51.96 \pm 18.96$ & & \\
\hline Female & $44.74 \pm 16.97$ & & \\
\hline \multicolumn{2}{|l|}{ BMI $\left(\mathrm{kg} / \mathrm{m}^{2}\right)$} & \multirow{4}{*}{2.952} & \multirow{4}{*}{0.056} \\
\hline$<18.5$ & $32.31 \pm 15.99$ & & \\
\hline $18.5 \sim 24$ & $50.93 \pm 17.67$ & & \\
\hline$>24$ & $48.27 \pm 18.79$ & & \\
\hline \multicolumn{2}{|l|}{ Employment status } & \multirow{4}{*}{0.081} & \multirow{4}{*}{0.923} \\
\hline In business & $47.93 \pm 18.07$ & & \\
\hline Retire & $49.17 \pm 18.60$ & & \\
\hline Unemployment & $50.16 \pm 20.71$ & & \\
\hline \multicolumn{2}{|l|}{ Average monthly income } & \multirow{4}{*}{5.867} & \multirow{4}{*}{0.004} \\
\hline Poor & $45.69 \pm 16.20$ & & \\
\hline Moderate & $45.68 \pm 17.84$ & & \\
\hline Good & $57.41 \pm 18.13$ & & \\
\hline \multicolumn{2}{|l|}{ Education level } & \multirow{5}{*}{0.939} & \multirow{5}{*}{0.424} \\
\hline Primary school and below & $47.28 \pm 16.22$ & & \\
\hline Junior middle school & $45.71 \pm 16.40$ & & \\
\hline Senior school or technical secondary school & $51.58 \pm 20.40$ & & \\
\hline College and above & $51.23 \pm 20.08$ & & \\
\hline \multicolumn{2}{|l|}{ Stage of disease } & \multirow{6}{*}{2.697} & \multirow{6}{*}{0.034} \\
\hline CKD stage 1 & $55.16 \pm 18.28$ & & \\
\hline CKD stage 2 & $53.09 \pm 17.16$ & & \\
\hline CKD stage 3 & $44.77 \pm 17.92$ & & \\
\hline CKD stage 4 & $46.28 \pm 18.08$ & & \\
\hline CKD stage 5 & $41.77 \pm 18.80$ & & \\
\hline \multicolumn{2}{|l|}{ Time of diagnosis (year) } & \multirow{4}{*}{0.093} & \multirow{4}{*}{0.911} \\
\hline$<1$ & $47.70 \pm 18.69$ & & \\
\hline $1 \sim 3$ & $48.68 \pm 16.94$ & & \\
\hline$>3$ & $49.33 \pm 18.81$ & & \\
\hline \multicolumn{2}{|l|}{ Comorbidity } & \multirow{4}{*}{0.636} & \\
\hline Cardiovascular diseases & $50.31 \pm 17.66$ & & 0531 \\
\hline Diabetes & $46.16 \pm 19.72$ & & 0.531 \\
\hline Gout & $48.89 \pm 18.69$ & & \\
\hline Daily physical activity(min) & & & \\
\hline$<30$ & $33.39 \pm 10.86$ & & \\
\hline $30 \sim 60$ & $57.36 \pm 13.16$ & 7.238 & 0.000 \\
\hline$>60$ & $67.46 \pm 11.39$ & & \\
\hline
\end{tabular}

CKD: Chronic Kidney Disease; BMI: Body Mass Index

Table 3: Comparison of CKD patient's exercise self-efficacy among the different socio-demographic and clinical characteristic 
Exercise self-efficacy was significant different among the different age $(t=1.988, P=0.049)$, different gender $(t=-2.310, P=0.022)$, average monthly income ( $F=5.867, P=004)$, stage of disease $(F=2.697, P=0.034)$, daily physical activity $(F=7.238, P=0.000$; Table 4$)$. The participants who lower 65 years had a significant higher level of exercise self-efficacy than those whose age more than 65 years. Compared to females, the male had higher confidence in exercising. Higher average monthly income participants had a higher level of exercise self-efficacy than the lower. The participants with more daily physical activity had better exercise self-efficacy than those with less. Participants with different stages also showed different levels of exercise self-efficacy (Table 3). No differences were found in BMI, employment status, education level, time of diagnosis and comorbidity (Table 3).

\section{Symptom burden and exercise self-efficacy}

The participants' average score on symptom burden was 19.43 \pm 6.51 (Range=11 46), indicating a heavier symptom burden. Person correlation analysis showed that there was a significant negative correlation between exercise self-efficacy and symptom burden in predialysis CKD patients, the correlation coefficient $r=-0.295(P<0.01)$.

\section{Predictive factors of exercise self-efficacy}

Stepwise multiple regressions were used to analyze the predicting factors of exercise self-efficacy. Variables showing statistically significant relationships with exercise self-efficacy were entered as the independent variables. These variables included age, gender, average monthly income, stage of disease, daily physical activity and symptom burdens, which were dummy coded before analysis (Tables 3 and 4).

The result showed that age, daily physical activity and symptom burden were significant predictors of exercise self-efficacy. These variables together explained $60.9 \%$ of the variance in exercise self-efficacy $(F=33.811 ; P<0.01$, Table 5$)$. The final regression equation is exercise self-efficacy: $11.392+\left(0.158^{\star}\right.$ age $)+\left(16.621^{\star}\right.$ daily physical activity $)-\left(0.429^{\star}\right.$ symptom burden $)$.

\begin{tabular}{|c|c|}
\hline Variable & Dummy coded \\
\hline Age & $<65=1 ; \geq 65=2$ \\
\hline Gender & Female $=1 ;$ Male $=2$ \\
\hline Monthly average income & Poor $=1$; Moderate $=2$; Good $=3$ \\
\hline Stage of disease & Stage $1=1$; Stage $2=2$; Stage $3=3$; Stage $4=4$; Stage $5=5$ \\
\hline Daily physical activity & $<30$ min $=1 ; 30 \sim 60$ min $=2 ;>60$ min $=3$ \\
\hline Symptom burden & Original value \\
\hline \multicolumn{2}{|c|}{ Table 4 : Dummy coded for categorical variables } \\
\hline
\end{tabular}

\begin{tabular}{|c|c|l|l|l|l|}
\hline Independent variables & B & SE & $\boldsymbol{\beta}$ & $\boldsymbol{t}$ value & $\boldsymbol{P}$ value \\
\hline Constant & 11.392 & 7.483 & - & 1.522 & 0.130 \\
\hline Age & 0.158 & 0.080 & 0.121 & 1.982 & $0.050^{*}$ \\
\hline Daily physical activity & 16.621 & 1.396 & 0.688 & 11.907 & 0.000 \\
\hline Symptom burden & -0.429 & 0.179 & -0.152 & -2.389 & 0.018 \\
\hline
\end{tabular}

1) SE, standard error. $\beta$, standardized regression coefficients

2) $R=0.781 ; R^{2}=0.609 ; F=33.811 ; P<0.01$

3) The result of $P$ value for age was 0.50 , so we incorporated it into the model

Table 5: Predictive factors of exercise self-efficacy in predialysis CKD patients

\section{Discussion}

\section{Predialysis CKD patients' exercise self-efficacy}

The result showed that the score of exercise self-efficacy of predialysis CKD patients was $48.85 \pm 18.42$, indicating a moderate level, which was higher than that of hemodialysis patients [40,(12.22, 58.33), medians and quartiles] [15]. As shown in Table 1 , the lower scores were item 1 "When you are feeling tired", item 17 "When you have other time commitments", item 3 "When you are experiencing bad weather", item 9 "When you feel physical discomfort when you exercise", Item 12 "When visitors are present". Therefore, there are many obstacles such as heavy disease burden and many objective factors in predialysis CKD patients during exercise. Wang et al. believe that before deciding whether to exercise regularly, individuals will not only measure the benefits of exercise but also subjectively evaluate whether they can adhere to exercise confidence. Self-efficacy plays a vital role in the relationship between exercise benefit and behavior change [16]. Bandura et al. also believe that self-efficacy is the key factor of individual motivation, which mediates the relationship between individual knowledge, skills and experience and subsequent behavioral changes [17]. Therefore, improve exercise self-efficacy is the prerequisite basis for the behavior change of predialysis CKD patients, to increase the amount of exercise. 
A qualitative study shows that fatigue, dyspnea, concern about the aggravation of illness caused by overexercising and bad weather were the main obstacles for predialysis CKD patients to participate in exercise [18]. Zelle et al. considered that healthcare providers should take into account behavioral and pathophysiological factors (ex. muscle mass or anemia), as well as objective factors, such as weather, traffic, etc. The interaction of these factors can lead to a reduction in the amount of exercise in predialysis CKD patients [7]. Jhamb et al. proposed that it is one of the common methods in behavioral change intervention to identify exercise-related barrriers and require healthcare providers and patients to formulate strategies to overcome these obstacles [19]. Therefore, understanding the patients' obstacles, motivation and confidence in exercise can enable us to adjust the intervention measures in time, and provide a reference basis for the implementation and development of behavior change programs for predialysis CKD patients who aim to promote exercise.

\section{Factors associated with exercise self-efficacy}

\section{Age}

Independent t-test showed that the lower the level of exercise self-efficacy in older patients, the score of exercise self-efficacy was $50.94 \pm 19.07$ in patients $<65$ years and $44.28 \pm 16.18$ in patients over 65 years $(P=0.049)$. Evidence indicated that the incidence of complications such as anemia, inflammation, malnutrition, and cardiovascular disease increases with age in predialysis patients, resulting in abnormal catabolism in individuals, including neuromuscular dysfunction, decreased exercise tolerance and reduced cardiopulmonary fitness [20]. The common symptoms of CKD patients, like muscle weakness, dyspnea, muscle spasm/stiffness, further aggravate this debilitating physical condition. As time goes by, the above abnormal metabolism may result in the limitation of motor function of patients, not only affect the activities of daily living, leading to a decline in health-related quality of life, but also further reduce the amount of exercise and form a vicious circle [7]. A cohort study found a decline in motor function at an early stage of kidney disease [21]. Unfortunately, a survey found that in the elderly population, low exercise is closely related to the faster decline of renal function, which may accelerate the process of dialysis [22]. Therefore, for elderly patients with CKD, earlier and/or multiple intervention strategies may be needed to prevent and/or alleviate the decline of motor function and increase their exercise self-efficacy.

\section{Daily physical activity}

One-way analyses of variance showed the patients with more daily activity had higher exercise self-efficacy, and the score of exercise selfefficacy with daily activity $<30$ min was $33.39 \pm 10.86$ while he scores of $30 \mathrm{~min}$ and $>60$ min were $57.36 \pm 13.16$ and $67.46 \pm 11.39$, respectively. In our study, daily physical activity is according to American College of Sports Medicine [23], assess the current physical activity levels of your patients can be quickly achieved using the Physical Activity Vital Sign (PAVS). The PAVS consists of just two questions, one is how many days per week do you engage in moderate to strenuous exercise, and another is how many minutes do you engage at this level. K/ DOQI guidelines recommend that CKD patients should exercise at least five times a week of moderate-intensity and last at least 30 min at a time [24]. But a recent study found that only $6.9 \%$ of CKD patients met the recommended daily activity levels [25]. This is mainly due to the lack of clear evidence-based exercise programs for CKD patients and guidance on how to implement exercise management in the population of $\mathrm{CKD}$ patients [26]. In most countries, there is a lack of adequate infrastructure and healthcare providers to promote exercise programs for CKD patients, which is not part of standard care for patients with kidney disease [26]. Therefore, doctors and nurses in the department of nephrology should discuss with patients the risks and benefits of exercise to clarify the adverse consequences associated with lack of exercise and inform patients that exercise is usually safe and feasible. Even moderate exercise can benefit.

\section{Symptom Burden}

Person correlation analysis showed that the higher the symptom burden, the lower the exercise self-efficacy. The kidney is involved in the regulation of multiple systems in the body, renal abnormalities can lead to multiple system disorders, leading to a variety of complications, including metabolic acidosis, anemia, mineral and bone disorder, hypertension. Nonspecific symptoms also include fatigue, anorexia, itching, nausea and vomiting, muscle spasm, edema and shortness of breath [27]. These symptoms promote each other and accelerate the decline of motor function in patients with CKD. Previous studies had shown that exercise can enhance cardiopulmonary endurance, regulate blood lipids, improve inflammation, and delay the progress of CKD [28]. Therefore, the challenge for renal health care is how to evaluate the diversity of symptoms and the level of exercise self-efficacy of CKD patients, to develop corresponding exercise prescription to reduce or even prevent the occurrence of disease symptoms.

\section{Study limitations}

This study has several limitations. First, the data were collected cross-sectionally, which makes it difficult to interpret any cause-effect relationship. In addition, we lack the evaluation of patients' drug and dietary restrictions, and the use of some antihypertensive drugs may affect patients' enthusiasm to participate in exercise, and malnutrition caused by protein intake may also be one of the factors for patients' refusal to exercise. Therefore, future studies can further explore the effects of drugs and diet on exercise self-efficacy in dialysis patients. Second, we enrolled patients in a single center that could decrease the generalisability of the results to other predialysis CKD patients. Third, caution is needed when expanding and interpreting our research results due to the lack of prior research measured with SEE. Despite these limitations, our study is the first study assessing exercise self-efficacy among predialysis CKD patients in the Shanghai area. The results of this study can be compared to other national or disease of exercise self-efficacy and used as basic data to develop exercise promotion programs for patients with CKD. 


\section{Implications for Practice}

The association between lack of exercise and poor prognosis is well verified for predialysis CKD patients. However, previous studies found that the exercise compliance of predialysis CKD patients was not ideal. Exercise self-efficacy is a main psychological factor affecting predialysis CKD patients to participate in exercise. This study showed that the exercise self-efficacy of predialysis CKD patients is in the moderate level, which is closely related to age, daily physical activity and symptom burden. Healthcare providers should carry out clinical work on the basis of comprehensive assessment of patients' needs, pay attention to the promoting effect of exercise self-efficacy on physical function rehabilitation, strengthen emotional support to patients, and enrich their coping strategies. Fundamentally improve patients' compliance with exercise.

\section{Conclusion}

The level of exercise self-efficacy in predialysis CKD patients is suboptimal. Age, daily physical activity and disease symptoms are the influencing factors of exercise self-efficacy were found to be the most problematic, which provides a starting point for health providers. When conducting exercise prescription, special attention should be paid to the elderly patients, and patients should be told to increase their daily physical activity, and those with more complications should pay attention to the management of their diseases to promote patients to increase the amount of exercise. Future research will require a longitudinal study and a cohort approach to obtain a deeper understanding of different factors with exercise self-efficacy in predialysis CKD patients.

\section{Acknowledgments}

We are especially grateful to all participants of the Nephrology of Longhua Hospital Affiliated to Shanghai University of Traditional Chinese Medicine for help with this study.

\section{Funding}

No funding was received for this study.

\section{Conflict of Interest}

The authors declare there were no conflicts of interest.

\section{References}

1. Chen N, Wang W, Huang Y, Shen P, Pei D, et al. (2009) Community-based study on CKD subjects and the associated risk factors. Nephrol Dial Transplant 24: 2117-23.

2. Ping H, Zhang X, Xing N (2012) Prevalence of chronic kidney disease in China. Lancet 380: 10.1016/S0140-6736(12)61213-6.

3. Gould DW, Graham-Brown MP, Watson EL, Viana JL, Smith AC (2014) Physiological benefits of exercise in pre-dialysis chronic kidney disease. Nephrology (Carlton) 19: 519-27.

4. Jha V, Garcia-Garcia G, Iseki K, Li Z, Naicker S, et al. (2013) Chronic kidney disease: global dimension and perspectives. Lancet 382: 260-72.

5. Wang F, Yang C, Long J, Zhao X, Tang W, et al. (2019) Executive summary for the 2015 Annual Data Report of the China Kidney Disease Network (CK-NET). Kidney Int 95: 501-5.

6. Hiraki K, Yasuda T, Hotta C, Izawa KP, Morio Y, et al. (2013) Decreased physical function in pre-dialysis patients with chronic kidney disease. Clin Exp Nephrol 17: 225-31.

7. Zelle DM, Klaassen G, van Adrichem E, Bakker SJ, Corpeleijn E, et al. (2017) Physical inactivity: a risk factor and target for intervention in renal care. Nat Rev Nephrol 13: 152-168.

8. Lee LL, Perng SJ, Ho CC, Hsu HM, Lau SC, et al. (2009) A preliminary reliability and validity study of the Chinese version of the self-efficacy for exercise scale for older adults. Int J Nurs Stud 46: 230-8.

9. Darawad MW, Khalil AA, Hamdan-Mansour AM, Nofal BM (2016) Perceived Exercise Self-Efficacy, Benefits and Barriers, and Commitment to a Plan for Exercise among Jordanians with Chronic Illnesses. Rehabil Nurs 41: 342-51.

10. Selzler AM, Rodgers WM, Berry TR, Stickland MK (2016) The importance of exercise self-efficacy for clinical outcomes in pulmonary rehabilitation. Rehabil Psychol 61: 380-8.

11. Foundation National Kidney (2002) K/DOQI clinical practice guidelines for chronic kidney disease: evaluation, classification, and stratification. Am J Kidney Dis 39: S1-266.

12. Brown SA, Tyrer FC, Clarke AL, Lloyd-Davies LH, Stein AG, et al. (2017) Symptom burden in patients with chronic kidney disease not requiring renal replacement therapy. Clin Kidney J 10: 788-96.

13. Tung WC, Gillett PA, Pattillo RE (2005) Applying the Transtheoretical Model to physical activity in family caregivers in Taiwan. Public Health Nurs 22: $299-310$.

14. Jagannathan R, Ziolkowski SL, Weber MB, Cobb J, Pham N, et al. (2018) Physical activity promotion for patients transitioning to dialysis using the "Exercise is Medicine" framework: a multi-center randomized pragmatic trial (EIM-CKD trial) protocol. BMC Nephrol 19: 230.

15. Guifang X, Yingjun Z, Huaihong Y, Xiaohong X (2018) Study on the influencing factors of exercise self_emcacy in maintenance hemodialysis patients. Chin J Blood Purif 17: 814-7.

16. Wang D, Ma Y (2014) An Analysis of Mediating Effect about Self-efficacy in Behavior Change Process of Physical Exercise. Journal of Xi'an Institute of Physical Education 2014: 242-9.

17. Bandura A, Adams NE (1977) Analysis of self-efficacy theory of behavioral change. Cognitive Therapy and Research 1: 287-310.

18. Clarke AL, Young HM, Hull KL, Hudson N, Burton JO, et al. (2015) Motivations and barriers to exercise in chronic kidney disease: a qualitative study. Nephrol Dial Transplant 30: 1885-92. 
19. Jhamb M, McNulty ML, Ingalsbe G, Childers JW, Schell J, et al. (2016) Knowledge, barriers and facilitators of exercise in dialysis patients: a qualitative study of patients, staff and nephrologists. BMC Nephrol 17: 192.

20. Odden MC (2010) Physical Functioning in Elderly Persons With Kidney Disease. Adv Chronic Kidney Dis 17: 348-57.

21. Odden MC, Whooley MA, Shlipak MG (2004) Association of chronic kidney disease and anemia with physical capacity: the heart and soul study. J Am Soc Nephrol 15: 2908-15.

22. Cohen RC, Katz R, Mozaffarian D, Dalrymple LS, Ian de Boer ID, et al. (2009) Physical activity and rapid decline in kidney function among older adults. Arch Intern Med 169: 2116-23.

23. Medicine ACOS (2019) Physical Activity Vital Sign[EB/OL], USA.

24. The National Kidney Foundation Kidney Disease Outcomes Quality Initiative (NKF KDOQI) (2005) K/DOQI Clinical Practice Guidelines for Cardiovascular Disease in Dialysis Patients, Guideline 14: Smoking, Physical Activity, and Psychological Factors, China.

25. Hayhurst WSG, Ahmed A (2015) Assessment of physical activity in patients with chronic kidney disease and renal replacement therapy. Springerplus 4: 536.

26. Williams AD, Fassett RG, Coombes JS (2014) Exercise in CKD: Why Is It important and How Should It Be Delivered?. Am J Kidney Dis 64: $329-31$.

27. Romagnani P, Remuzzi G, Glassock R, Levin A, Jager KJ et al. (2017) Chronic kidney disease. Nat Rev Dis Primers 3: 17088.

28. Roshanravan B, Gamboa J, Wilund K (2017) Exercise and CKD: Skeletal Muscle Dysfunction and Practical Application of Exercise to Prevent and Treat Physical Impairments in CKD. Am J Kidney Dis 69: 837-52.

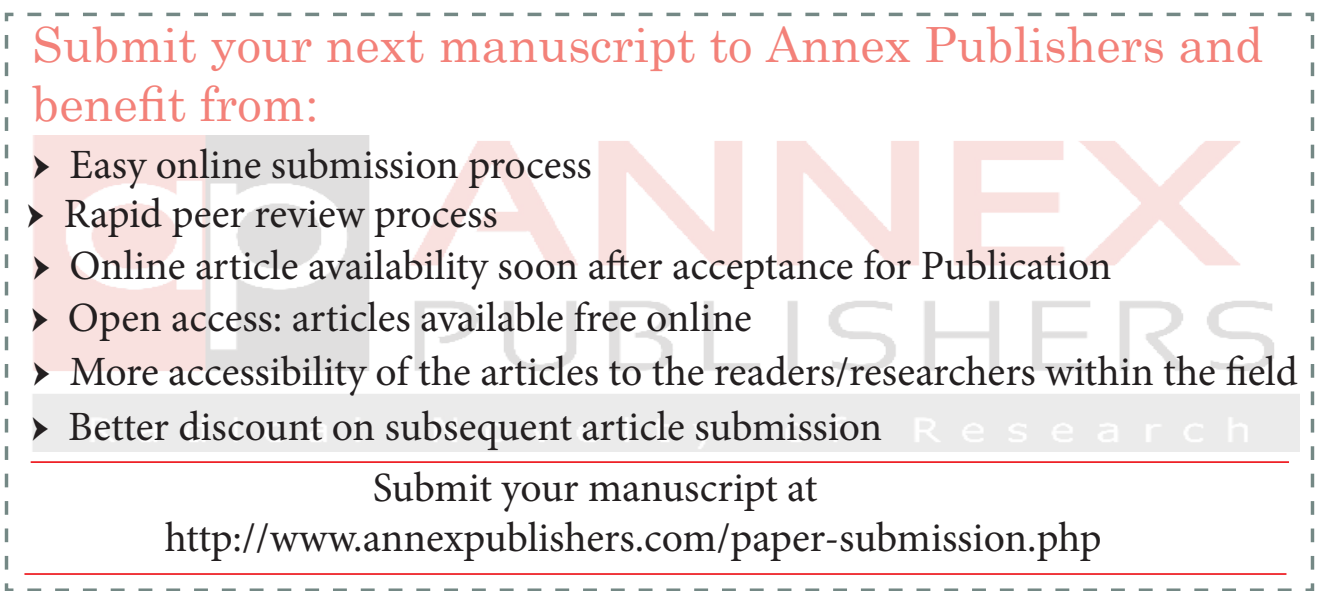

